\title{
Integrated Modelica Model and Model Predictive Control of a Terraced House Using IDEAS
}

\author{
Filip Jorissen $^{1} \quad$ Lieve Helsen $^{1,2}$ \\ ${ }^{1}$ Mechanical Engineering, KU Leuven, Belgium, \{filip.jorissen, lieve.helsen\}@kuleuven.be \\ ${ }^{2}$ EnergyVille, Belgium
}

\begin{abstract}
Modelica has been used extensively within the Thermal System Simulation (The SySi) research group at KU Leuven to simulate and optimize the control and design of building energy systems. Within this scope, the open source Modelica library IDEAS has been developed and papers have been published that explain how IDEAS can be used to develop fast simulation models and MPC. This paper presents an open-source simulation model of a terraced house for which these earlier presented guidelines are applied and for which MPC results are made available. A full-year simulation of the nine-zones model takes four minutes and energy savings of $12.8 \%$ are reported compared to a current-practice rule-based controller, although MPC has thermal comfort violations of up to $0.4 \mathrm{~K}$.
\end{abstract}

Keywords: IDEAS, Model Predictive Control, TACO, Building Energy Simulation

\section{Introduction}

Building space heating and HVAC account for $15 \%$ of the world final energy use (International Energy Agency, 2015). Therefore, according to the European Union's Directive 2010/31/EN (European Parliament, 2010), an increasing effort is spent at increasing buildings efficiency by improving their insulation level, by installing HVAC systems with a high primary energy efficiency (e.g. a combination of heat pump and floor heating or concrete core activation), and by increasing the share of renewable energy sources in buildings. These systems increase the thermal time constants of the building and introduce a rapidly changing collection of devices into the built environment. Efficient design and operation of buildings therefore calls for a dynamic simulation tool that can follow rapidly changing trends. Furthermore, further system integration with district heating systems and electrical distribution networks calls for a tool that is not limited to a fixed set of disciplines. 'Traditional' Building Energy Simulation (BES) tools often only offer a limited set of models and can be difficult to extend. Furthermore, their algorithms often tightly integrate equations and algorithms for solving them into the same code (Wetter et al., 2016; Wetter, 2009; Wetter et al., 2015), which complicates the maintenance of such codes. Furthermore, extracting model equations is usually not supported.
Modelica thus has some clear advantages over these BES tools. Firstly, Modelica is not limited in terms of what models can be integrated. Secondly, Modelica treats the model equations and algorithms for solving them separately. The same set of equations can thus be coupled to different solvers. This includes solvers both for simulation and optimization. Since the model equations are available, they can be differentiated automatically, which enables the use of highly efficient derivative-based optimization algorithms.

For these reasons, the Thermal Systems Simulation (The SySi) research group at KU Leuven has co-developed the open-source Modelica library IDEAS (Jorissen et al., 2018c) together with the Building physics department and the company 3E since 2010. One of the main motivations for our continued development of IDEAS, is to use the building models for optimal design and control applications, where the availability of the model equations poses a crucial advantage. The development of IDEAS is part of the IBPSA project 1, the successor of the IEA EBC Annex 60 (Wetter and van Treeck, 2017), which coordinates the development of IDEAS with the Modelica libraries Buildings (Wetter et al., 2014), AixLib (Müller et al., 2016) and BuildingSystems (Nytsch-Geusen et al., 2013). Our research focusses in particular on Model Predictive Control (MPC). Based on IDEAS, two main white-box approaches for MPC have been developed so far.

Firstly, the model can be implemented using component models from the package IDEAS. LIDEAS, which contains component models that support linearisation. This way the building envelope can be exported in the state space form

$$
\frac{\mathrm{d} x(t)}{\mathrm{d} t}=A x(t)+B u(t) .
$$

Where variables $x(t)$ are state variables and variables $u(t)$ are the boundary conditions of the building such as the solar irradiation that enters through each window. Time series data for the vector $u(t)$ is also generated using IDEAS. The model can thus be exported to other frameworks such as python or Matlab. The component models, templates and a minimum working example required for using LIDEAS have recently been made open source and can now be found in IDEAS. LIDEAS. For more de- 
tails with respect to this linearisation toolchain we refer the reader to Picard et al. (2015). This methodology can only be used to export linear models and as such its functionality is limited to linear problems, although non-linear equations can be added to the exported linear model manually.

Secondly, to overcome these hurdles, a non-linear Toolchain for Automated Control and Optimization (TACO) has recently been developed by Jorissen et al. (2018b). TACO interprets a Modelica model using the JModelica framework (Åkesson et al., 2010) and automatically translates the model into an efficient optimization code that is implemented using CasADi (Andersson et al., In Press, 2018). The goal of TACO is to significantly reduce the engineering overhead required for developing MPCs for building applications by leveraging object oriented Modelica models. Jorissen et al. (2018a) describe the detailed Modelica model of an office building and a comparison with measured data. An MPC has been developed for this model by Jorissen (2018). In simulations the operational cost of the building was reduced by more than $50 \%$, however thermal depletion of the ground by passive cooling was not accounted for.

These applications demonstrate the potential of Modelica and MPC for building applications. Since Modelica is a general-purpose modelling language, multiple problem formulations are possible and multiple solvers can be chosen by the user. Both affect the model computation time and robustness for simulations and optimizations. Our models combine many state variables in the building envelope model with non-linear equations and algebraic loops in the Heating, Ventilation and Air Conditioning (HVAC) models, and discrete equations in the building control. For the simulation of large models this combination can lead to long computation times. Jorissen et al. (2015) and Jorissen et al. (2018d) describe how models can be manipulated to speed up computations. Furthermore, we propose to use explicit time integrators such as Euler integration since the computation time of Euler integration scales better with the number of state variables than for implicit solvers. These tips are applied by Jorissen et al. (2018a) on the model of a 32-zones office building.

These guidelines for implementing computationally efficient building models using Modelica may however be difficult to apply by IDEAS users since a clear example model is not available. Similarly, unexperienced Modelica users may not understand how these integrated building models can be structured. This limits the usability of IDEAS. This paper therefore presents a Modelica example model of a 9 zones terraced house building in Modelica. The guidelines for increasing the simulation speed are applied and explained. Furthermore, the performance of an MPC controller for this model is compared to a rule-based controller (RBC). This comparison can be extended in the future within the scope of the IBPSA project 1, which aims to develop BOPTEST, a set of benchmarks for comparing advanced building controllers such as MPC. Both the model and the optimal control trajectories from the MPC are included in the IDEAS library as of release 2.1 such that they can serve as a case study model or benchmark for other research applications.

This paper is structured as follows. Section 2 describes the building that is modelled and Section 3 explains how this building is modelled using components from the IDEAS library. Section 4 then explains how the guidelines from earlier work are applied to this example model and presents the resulting computation speed. Section 5 compares an MPC based on this model to the RBC implementation that is included with the model. Conclusions are presented in Section 6.

\section{Building Description}

This paper presents the IDEAS model of a real terraced house that consists of three floors and an attic. The building layout is sketched in Figure 1. The ground floor consists of 1) a living room and a hallway with a combined surface area of $4.6 \mathrm{~m} \times 8 \mathrm{~m}$ in the front of the building and 2) a dining room, kitchen and glazed veranda behind the main building, which have a combined ground surface area of $4.6 \mathrm{~m} \times 5 \mathrm{~m}$. The first floor consists of a hallway $(1.6 \mathrm{~m} \mathrm{x} 4 \mathrm{~m})$, bathroom $(3 \mathrm{~m} \mathrm{x} 4 \mathrm{~m})$ and bedroom (4.6 $\mathrm{m} \times 4 \mathrm{~m})$. The top floor consists of a bedroom $(4.6 \mathrm{~m} \times 4$ $\mathrm{m})$ underneath a mansard roof, and an office (4.6 $\mathrm{m} \mathrm{x} 4 \mathrm{~m}$ ) underneath a gabble roof, which contains a Velux window of $1.2 \mathrm{~m}^{2}$. Both parts of the roof are insulated with $12 \mathrm{~cm}$ of polyurethane, $5 \mathrm{~cm}$ of glass wool and are finished with plasterboard of $12.5 \mathrm{~mm}$.

The building was constructed around 1926 and its façade consists of uninsulated brick walls without cavity and a thickness of approximately $28 \mathrm{~cm}$. The front $4 \mathrm{~m}$ of the building has a cellar, whose ceiling is uninsulated. The ground floor consists of parquet on tiles on solid ground, while the other floors consist of fiberboard or wooden floor board on wooden beams. The ceilings below the floors are finished using plasterboard, which is attached to a metal frame. An acoustic insulation layer of $5 \mathrm{~cm}$ lies on top of this frame. The building windows consist of PVC frames with triple glazing. A vertical technical shaft runs through center of the building, which contains the heating system pipes and collectors and the ventilation ducts.

The building is heated using a gas boiler of $30 \mathrm{~kW}$ (Bulex Thermomaster T30/35). Radiators supply heat to each of the rooms except the dining room, porch and kitchen. The radiators are connected to a common collector for each floor using alupex tubes. The collectors are connected in series to the boiler. The boiler is on-off controlled using a Bulex Exacontrol E7C thermostat in the living room. A ventilation unit (Brink Renovent Excellent $300+$ ) supplies air to the bedrooms, office and living room 


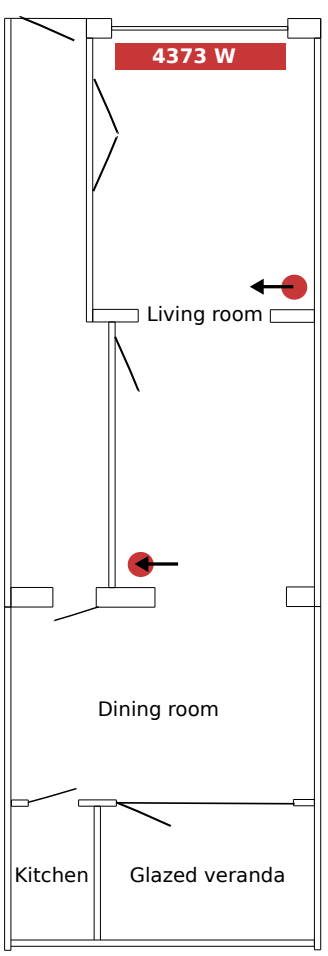

Ground floor
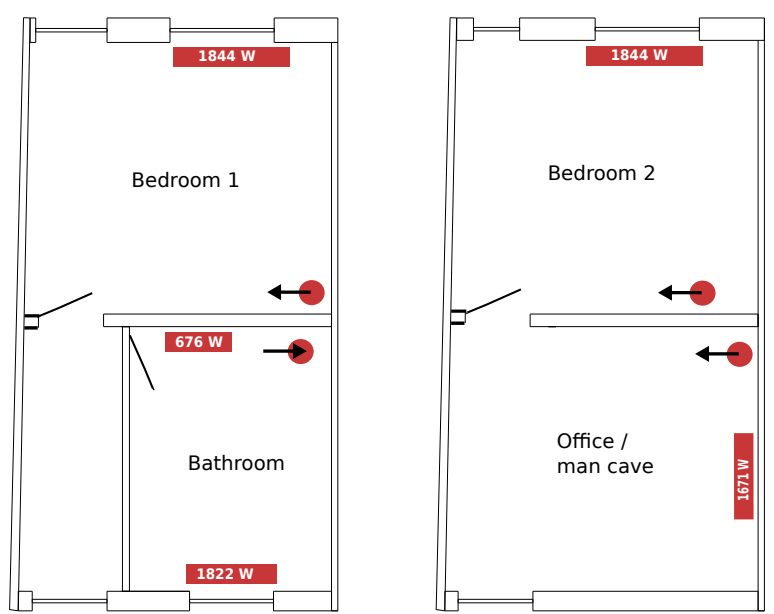

Legend

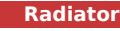

$\longrightarrow$ Ventilation supply

Ventilation exhaust

First floor

\section{Second floor}

Figure 1. Sketch of the building layout and the location of radiators and vents.

and extracts air in the bathroom and living room. The nominal air flow rate of the unit is $300 \mathrm{~m}^{3} / \mathrm{h}$, for which heat is recovered with an efficiency of approximately 75 $\%$. The heat exchanger is automatically bypassed during summer, which is not modelled yet. The ventilation unit operates at a fixed flow rate of $70 \mathrm{~m}^{3} / \mathrm{h}$.

\section{Model Description}

An integrated Modelica model of this building is created using the IDEAS library (Jorissen et al., 2018c). The model consists of four parts:

1. The building envelope, which consists of zones, walls and windows.

2. The heating system, which consists of a pump, heater, pipes, radiators and thermostatic valves.

3. The ventilation system, which consists of fans, ducts, two bypass valves and a heat exchanger.

4. The thermostat.

Each of these parts is illustrated in Figure 2. The model is part of the IDEAS library and can be found in the package IDEAS. Examples.PPD12.

\subsection{Ventilation System}

The top of Figure 2 illustrates the ventilation system, which consists of two fans, two bypasses, a heat exchanger and ducts that are connected to the zones. Ducts are modelled using the component IDEAS.Fluid.FixedResistances. Junction. The pressure drop of each branch of the junction is estimated from the design flow rate of the system and the diameter and free area fraction of the vents. The pressure drop of the ducts is neglected. This results in nominal pressure drops in the order of $500 \mathrm{~Pa}$ for a flow rate of 0.1 $\mathrm{kg} / \mathrm{s}$.

The model IDEAS.Fluid.Movers. FlowControlled_m_flow from the IBPSA library (Wetter, 2013) prescribes the flow rate of the fans. The total pressure drop is thus computed from the fluid flow network, which is in turn used to compute the fan electrical power use. A fixed fan efficiency of $23.75 \%$ is assumed, which coincides with the measured efficiency at nominal flow rate.

The model IDEAS.Fluid.HeatExchangers. ConstantEffectiveness computes how much heat is recovered. It assumes a fixed heat exchange effectivity. Ideal dampers, without pressure drop, are used to model the bypasses.

As indicated in Figure 1, air is injected in some rooms, while air is extracted from other rooms. Manual connections are added to allow air exchange between rooms such that mass is conserved. In the future this functionality will be integrated into IDEAS such that these connections need not be added by the user. 


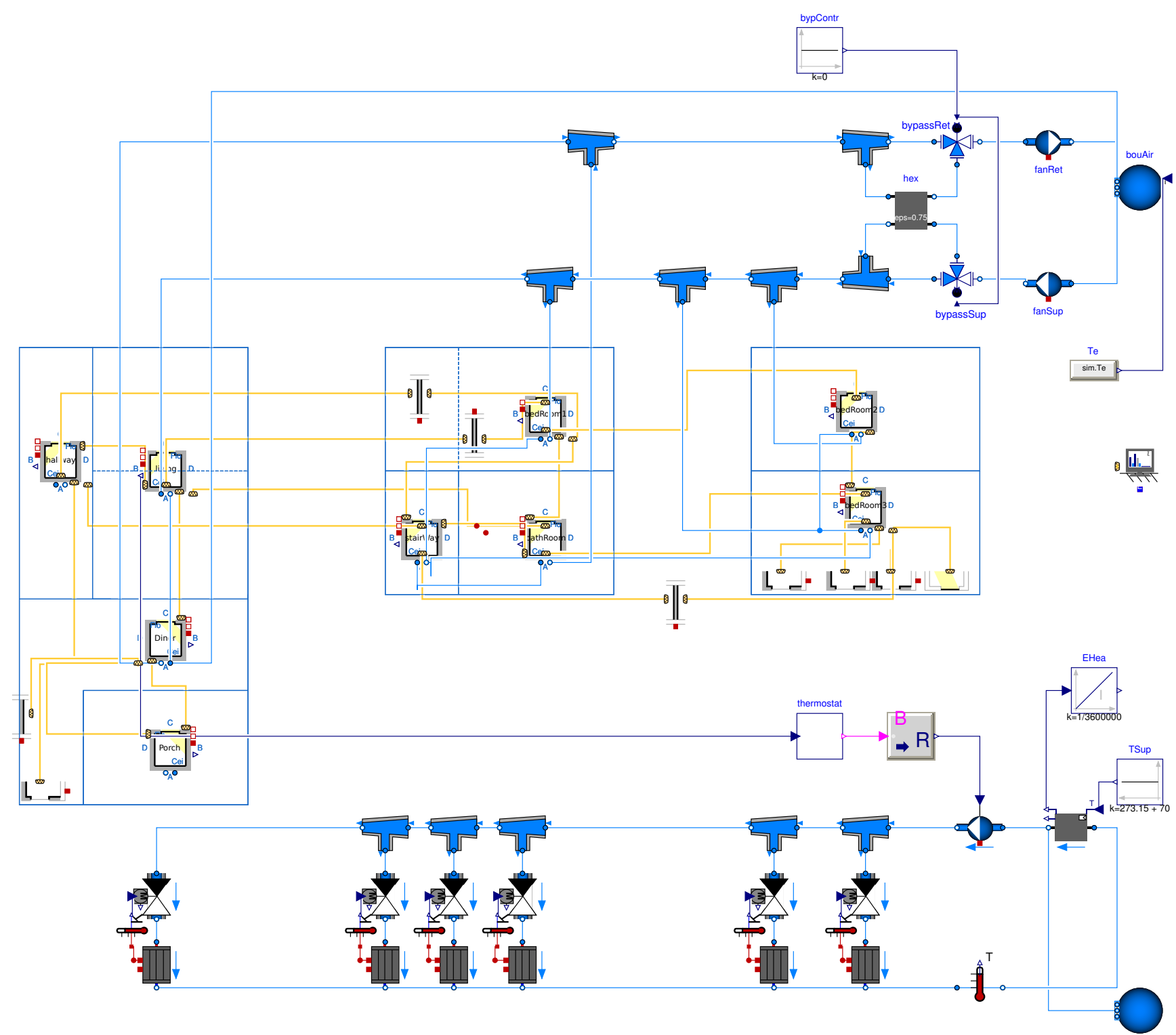

Figure 2. Illustration of the Modelica model of the building. The top includes the ventilation, which consists of two fans, a heat exchanger, ducts and two bypasses. The middle contains the building envelope model, which consists of walls, windows and zone templates, which integrate more walls and windows for a rectangular zone. The bottom shows the heating system, which consists of a heater, pump, pipes, radiators and thermostatic valves. In between middle and bottom the thermostat can be found. 


\subsection{Building Envelope}

The middle of Figure 2 represents the building envelope, which is modelled using components from IDEAS.Buildings. The main functionality of these components was described by (Jorissen et al., 2018c). Most notably, the model IDEAS.Buildings. Components. RectangularzoneTemplate is used to define the zones, their parameters and interconnections. Interior walls, external walls, roof and windows are included in the zone template for each orientation. When the zone geometry is not simply rectangular, or when a floor has to be split into two parts, additional surfaces are added manually. See e.g. two separate floor models are used to model the floor of bedroom 1 since it is both above the living room and the hallway. The radiators and ventilation system are also connected to the zones but these connections are hidden in Figure 2. The surface (walls, windows, etc.) parameters consist of the surface dimensions, orientations and its structure, which is defined using records such as illustrated in listing 1 .

Listing 1. Example use of a record to define the material layers of a ceiling

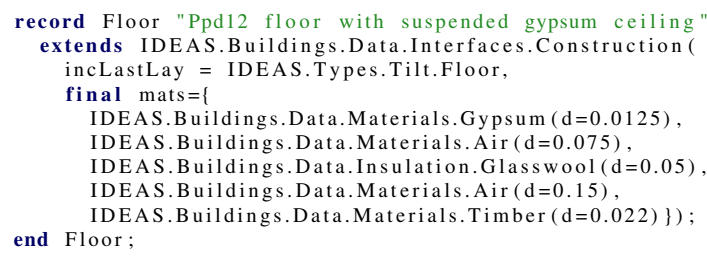

The wall opening between the living room and the dining room is modelled using the recently introduced option in the InternalWall model to model a 'cavity' or door. This model approximates the buoyancy-driven advection between zones using the zone temperature differences, the ideal gas law and Bernoulli's principle. We assume that all doors are closed, i.e. they are not modelled using the door model option. Furthermore, we assume that the common walls with the neighbours are adiabatic.

\subsection{Heating System}

The bottom of Figure 2 illustrates the heating system model. It consists of six radiators, a pump and an ideal heater. Radiator thermal powers are indicated in Figure 1. The pump head is unknown and is assumed to be $100 \mathrm{kPa}$. The ideal heater model IDEAS.Fluid. HeatExchangers. PrescribedOutlet is used, which supplies a prescribed temperature set point unless this implies that a thermal power of more than $30 \mathrm{~kW}$ or less than $0 \mathrm{~kW}$ is supplied. The boiler efficiency is computed as a function of the return water temperature using the polynomial fit to the data that is provided in a temperature range of $25^{\circ} \mathrm{C}$ to $65^{\circ} \mathrm{C}$. The slope at the end of the range is extrapolated up to $75^{\circ} \mathrm{C}$. The resulting efficiency is implemented as in Listing 2.
Listing 2. Efficiency implementation of the boiler Modelica.SIunits.Efficiency eta $=\{-6.017763 \mathrm{e}-11,2.130271 \mathrm{e}-8$,
$-3.058709 \mathrm{e}-6,2.266453 \mathrm{e}-4, \quad-9.048470 \mathrm{e}-3,1.805752 \mathrm{e}-1$, $-4.540036 \mathrm{e}-1\} *\left\{\operatorname{TRet}^{\wedge}(6-\mathrm{i})\right.$ for $\mathrm{i}$ in $\left.0: 6\right\}$;

Radiators are modelled using the model RadiatorEN442_2. Each radiator has a thermostatic valve, which is modelled using the model TwoWayTRV, which consists of a valve with a linear opening characteristic that is controlled by a proportional controller with a fixed temperature set point and a proportional band of $2 \mathrm{~K}$. The proportional controller is implemented such that the valve is closed when the temperature set point is reached. The set point equals $30{ }^{\circ} \mathrm{C}$ for the radiator in the living room, since the thermostat is mounted in this room. The remaining radiators have a set point of $21^{\circ} \mathrm{C}$. The pressure drop of the pipes is computed using their dimensions.

\subsection{Thermostat}

The thermostat is a custom implementation, which turns the boiler on or off. Since the supply temperature is not actively controlled, the heater supply water temperature is fixed to $70{ }^{\circ} \mathrm{C}$. The thermostat operates on a schedule. The temperature set point is $21{ }^{\circ} \mathrm{C}$ from 7:00 to 9:00 and from 18:00 to 23:00 on week days and from 7:00 to 23:00 on weekend days and $16{ }^{\circ} \mathrm{C}$ otherwise. Note however that the thermostat is activated one hour earlier to ensure that the building has heated up in time. Since the thermostat is mounted in the living room, the thermostat uses the living room temperature as an input. Furthermore, it implements a hysteresis controller with a hysteresis band of $1 \mathrm{~K}$ above the set point temperature. When enabled, the boiler pump head is set to 1 bar.

\section{Computational Aspects}

We now discuss some Modelica-related implementation details that affect the computation time.

\subsection{Computation Time}

As described by Jorissen et al. (2015), implicit integrators are not well suited for simulating models with many states and, depending on the model size, explicit integrators such as Euler integration may be more suited. Our model has about 330 state variables. Therefore, the model parameters have been chosen to avoid small time constants by considering fast processes to be steady state, such that the model can be simulated using (explicit) Euler integration. We now explain for each part of the model how this was done.

\subsection{Envelope Model Configuration}

Jorissen et al. (2018c) describe in detail how the models in IDEAS.Buildings have been adjusted to speed up computations. I.e. fast dynamics in the window glazing are lumped into a single thermal capacitor and non-linear algebraic loops are avoided by choosing an appropriate discretisation scheme of the heat conduction equations. 
Furthermore, we configure the massDynamics of the zone models to Steadystate, which implements an incompressible air model, which thus avoids a state variable for the zone air pressure.

\subsection{Ventilation System Configuration}

In the ventilation system we avoid the generation of algebraic loops that solve for enthalpy by using the portFlowDirection_* variables. Furthermore, the energyDynamics and massDynamics of the junctions, fans and bypasses are set to SteadyState, which avoids more states with a small time constant. Similarly, the input filter of the fans is removed.

\subsection{Heating System Configuration}

In the heating system, the used water medium is already incompressible by default, such that we need not set massDynamics. The valve filters are not removed since they react already sufficiently slowly. However, the junctions energyDynamics are set to Steadystate. The radiator energy dynamics are not neglected. However, the number of elements of the radiator is reduced from the default value of 5 to 3 , such that the radiator segment volumes are sufficiently large to avoid instabilities when the radiator mass flow rate is large. Furthermore, the series pressure drops of the thermostatic valve, the radiator and the pipes are merged into a single pressure drop equation by using the parameter dpFixed_nominal of the valve model. The pressure drop of the pipes between the collectors of each floor are neglected since their internal diameter is $20 \mathrm{~mm}$ instead of $12 \mathrm{~mm}$, which causes pressure drops that are 7.7 times smaller for the same mass flow rate, moreover these pipes are shorter. This causes the earlier mentioned series connections to be in parallel with respect to each other, meaning that they have the same pressure drop. This can be exploited by setting from_dp=True in the valve models, since then a single iteration variable can be used to solve the resulting algebraic loop (Jorissen et al., 2018d). For a more detailed discussion and motivation for these configurations and simplifications we refer the reader to Jorissen et al. (2018d) and Jorissen et al. (2015).

\subsection{Computation Time Results}

Using this configuration, which also speeds up Dassl, the model can be evaluated at a speed 156000 times faster than real time using explicit Euler integration with a fixed step size of $15 \mathrm{~s}$ and 44000 times faster than real time using Dassl with a tolerance of $10^{-4}{ }^{1}$ Simulating a full year thus requires 4 minutes. Euler has an error of $0.14 \%$ on the total computed energy use when comparing to LSodar with a tolerance of $10^{-6}$, while Dassl has an error of $0.017 \%$.

${ }^{1}$ On a Macbook pro with $2.7 \mathrm{GHz}$ i7-6820HQ processor, using Dymola 2019 with option Evaluate=true and virtual machine software Parallels 11 running Ubuntu 14.04.

\section{Application of Model Predictive Control}

While IDEAS was originally designed as a Modelica library for building and district energy simulation, the models are now also suited for optimization applications such as MPC. Using TACO, the presented model can be translated into an MPC controller by performing only minor modifications such as linearising the building envelope heat transfer equations (Jorissen, 2018, Appendix A).

As a demonstration, an MPC controller was developed that minimizes

$$
J(t)=P_{\text {fan }, \text { sup }}(t)+P_{\text {fan }, \text { ret }}(t)+0.25 \dot{Q}(t)
$$

where $P_{f a n}(t)$ is the electrical power use of the fans and $\dot{Q}(t)$ is the thermal power use of the heater. A weighting factor of 0.25 is used since gas is about four times less expensive than electricity in Belgium. Furthermore, the living room operative temperature is lower bounded to $21^{\circ} \mathrm{C}$ during the schedule indicated in Section 3.4. We optimize the heater supply water temperature, which is upper bounded to $75{ }^{\circ} \mathrm{C}$ and the fan mass flow rates, which are lower bounded to their set point value of $70 \mathrm{~m}^{3} / \mathrm{h}$ and upper bounded to the nominal value of $300 \mathrm{~m}^{3} / \mathrm{h}$. The bypasses are closed and the pump is always enabled. The resulting controller is coupled to the simulation model and is operated for a full year in a closed loop simulation. The optimal control results are stored in a csv file and are included as a benchmark in the model IDEAS. Examples. PPD12.VentilationMPC.

$$
\text { A comparison with RBC (see }
$$

IDEAS.Examples.PPD12.VentilationRBC),

which operates on the same temperature set point and using the same schedule, is shown in Figure 3. The figure shows that MPC is unable to satisfy the comfort constraint, which is caused by model mismatch between the MPC model and the simulation model. For instance, the building envelope convective heat transfer equations are linearized for the MPC, which causes the heat convection coefficients to be overestimated. The average comfort violation of MPC for the living room peaks at about $0.4 \mathrm{~K}$.

MPC often uses much lower supply water temperatures than $\mathrm{RBC}$, of about $45^{\circ} \mathrm{C}$, which is reflected in a smoother heating profile. This is clearly visible in the thermal power of the heater. Furthermore, Figure 5 shows smoother temperature profiles for zones that are controlled using a thermostatic radiator valve, which heat the rooms more than required due to the thermal inertia of the radiators and the zone air temperature. The total energy use of RBC is $6590 \mathrm{kWh}$ while MPC uses only $5749 \mathrm{kWh}$. This implies a final energy use reduction of $12.8 \%$. While these are modest energy savings, this is to be expected for badly insulated buildings with simple heating systems, 

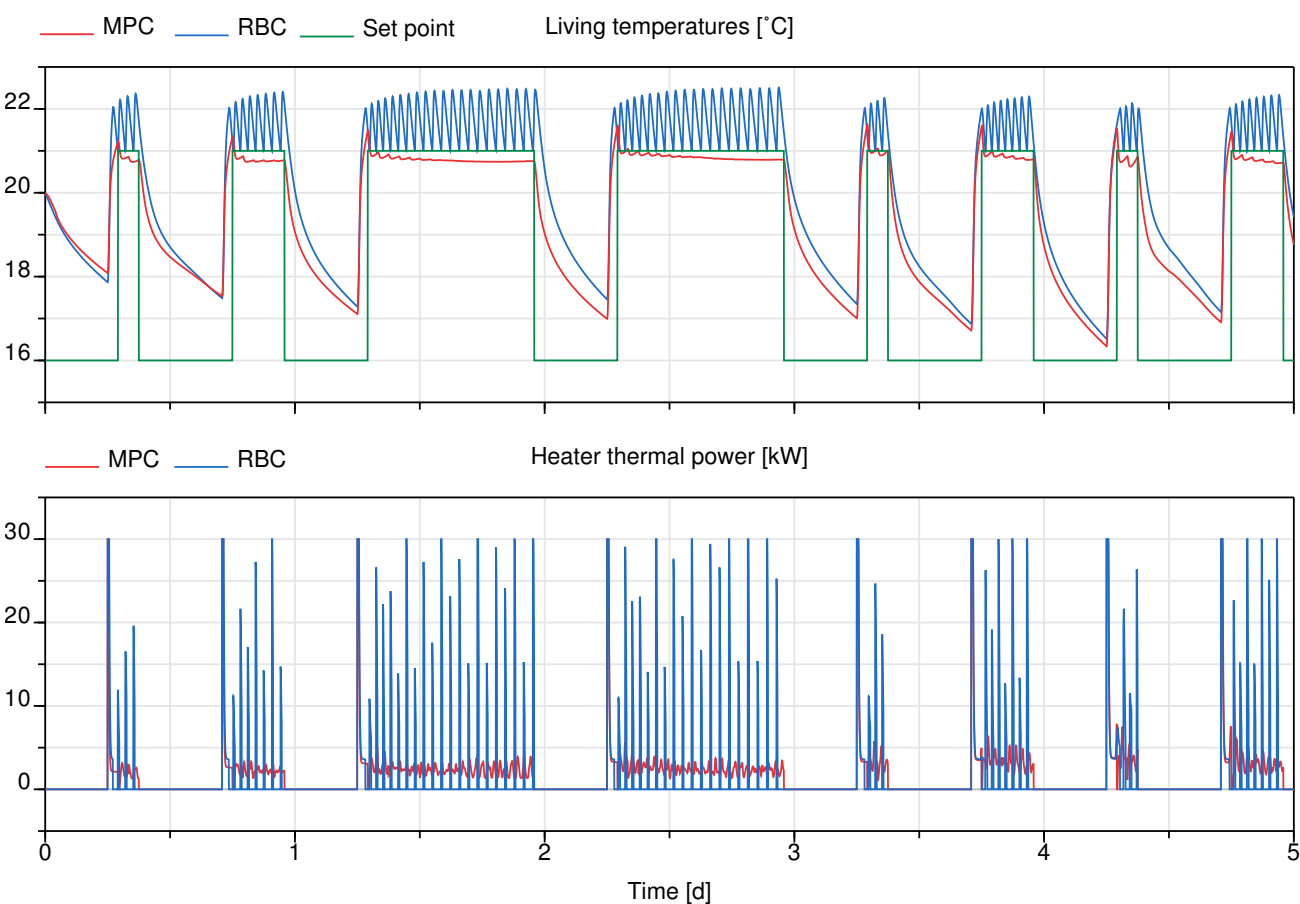

Figure 3. Comparison of zone temperature and thermal power results for RBC and MPC

which have only limited flexibility that can be exploited by MPC. These energy savings are only partly explained by the comfort violations of MPC, since lowering the RBC temperature set point by $1 \mathrm{~K}$ for the whole year results in an energy use of $6212 \mathrm{kWh}$.

The MPC uses control intervals of one hour such that it is forced to start heating one hour in advance in order to satisfy the comfort constraint. If smaller control intervals were used, the pre-heating could be postponed which would lead to larger energy savings.

The model also computes the return water temperature dependent gas energy use. The MPC has a lower supply water temperature so it is unexpected that the average heat generation efficiency of MPC is in fact lower than that of RBC. The gas energy use for MPC is $6317 \mathrm{kWh}$ instead of $7218 \mathrm{kWh}$ (only $12.5 \%$ reduction). This is at least partly caused by the fact that the pulsed behaviour of $\mathrm{RBC}$ causes it to have relatively low return water temperatures for the short period of time when the boiler is active, which increases the efficiency. During a winter period, for $\mathrm{RBC}$ the return water temperatures rises from about $30^{\circ} \mathrm{C}$ to $50{ }^{\circ} \mathrm{C}$, while the MPC has a more constant return water temperature of about $40^{\circ} \mathrm{C}$, as illustrated in Figure 4 . Note that the largest heat production for RBC occurs at the lower range of the return water temperatures. This shows the importance of integrated system simulations for making detailed analyses. Note however, that these results may no longer hold when more detailed convection correlations and a more detailed boiler efficiency computation are used.

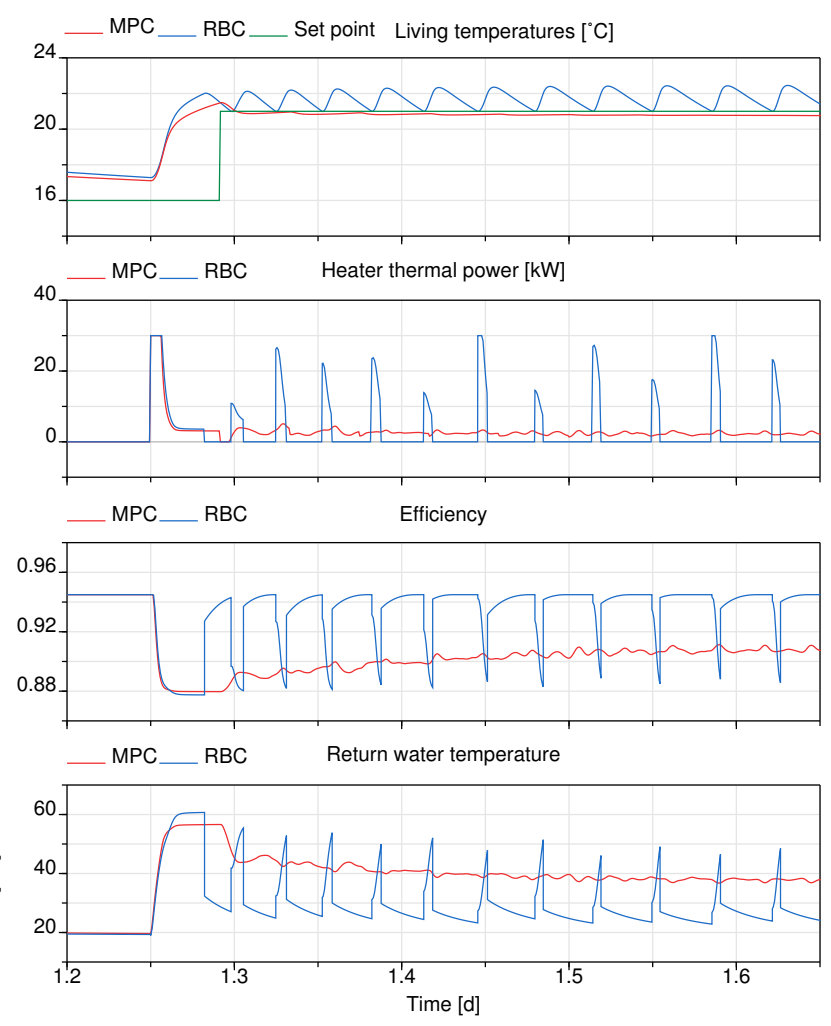

Figure 4. Comparison of heater efficiency for RBC and MPC 

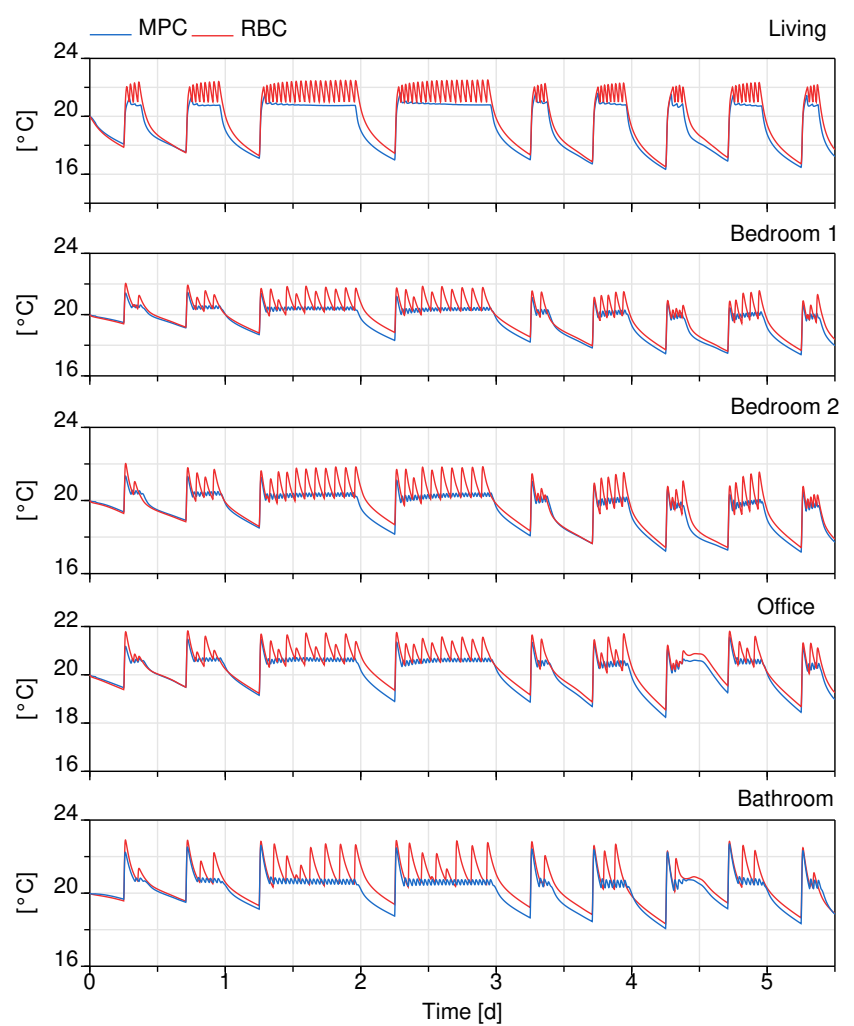

Figure 5. All zone temperatures for RBC and MPC

\section{Conclusion}

Modelica and open-source libraries such as IDEAS can have large added value in the building energy simulation and optimization community. This paper applies formerly published guidelines for efficient model development using IDEAS and implementation details to reduce computation time to the example model of a terraced house. The example is provided open-source and thus provides Modelica and IDEAS users with a clear example of how to use IDEAS for integrated building energy simulations. The building is first described, after which the model implementation and computational aspects are explained. The model simulation time is 4 minutes for one year. Furthermore, a white-box Model Predictive Controller (MPC) is generated for the model using TACO, a Toolchain for Automated Control and Optimization. MPC uses $12.8 \%$ less energy than rule-based control, which is partly caused by the lower supply water temperatures that generate a smoother heating profile. While these are modest energy savings, larger energy savings are typically obtained for more complex buildings with longer time constants than this uninsulated building with a simple heating system. The model and MPC results are available in the IDEAS library in the package IDEAS.Examples.PPD12 and can thus serve as a starting point for other research. Future work will focus on validating the model and on expanding the heating system complexity and thus its control options. More complex benchmarks will be developed within the frame of BOPTEST development in IBPSA project 1.

\section{Acknowledgements}

This project has received funding from the European Union's Horizon 2020 research and innovation program under grant agreement No 723649. The original project acronym is "MPC-.GT".

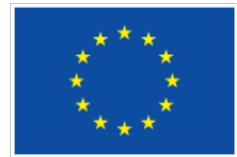

Co-funded by the Horizon 2020 programme of the European Union

This work emerged from the IBPSA Project 1 , an international project conducted under the umbrella of the International Building Performance Simulation Association (IBPSA). Project 1 will develop and demonstrate a BIM/GIS and Modelica Framework for building and community energy system design and operation.

\section{References}

J. Åkesson, K.-E. Årzén, M. Gäfvert, T. Bergdahl, and H. Tummescheit. Modeling and optimization with Optimica and JModelica.org - Languages and tools for solving large-scale dynamic optimization problems. Computers \& Chemical Engineering, 34(11):1737-1749, 2010. doi:10.1016/j.compchemeng.2009.11.011.

J. A. E. Andersson, J. Gillis, G. Horn, J. B. Rawlings, and M. Diehl. CasADi - A software framework for nonlinear optimization and optimal control. Mathematical Programming Computation, In Press, 2018.

European Parliament. Directive 2010/31/EU of the european parliament and of the council of 19 may 2010 on the energy performance of buildings (recast). Official Journal of the European Union, 18(06), 2010.

International Energy Agency. World energy outlook 2015. Technical report, 2015.

F. Jorissen. Toolchain for Optimal Control and Design of Energy Systems in Buildings. Phd thesis, Arenberg Doctoral School, KU Leuven, April 2018.

F. Jorissen, M. Wetter, and L. Helsen. Simulation Speed Analysis and Improvements of Modelica Models for Building Energy Simulation. In 11th International Modelica Conference, pages 59-69, Paris, 2015. doi:10.3384/ecp1511859.

F. Jorissen, W. Boydens, and L. Helsen. Implementation and Verification of the Integrated Envelope, HVAC and Controller Model of the Solarwind Office Building in Modelica. Journal of Building Performance Simulation, 2018a. doi:10.1080/19401493.2018.1544277. Published on line.

F. Jorissen, W. Boydens, and L. Helsen. TACO, an Automated Toolchain for Model Predictive Control of Building Systems: Implementation and Verification. Journal of Building Performance Simulation, 12(2):180-192, 2018b. doi:10.1080/19401493.2018.1498537.

F. Jorissen, G. Reynders, R. Baetens, D. Picard, D. Saelens, and L. Helsen. Implementation and Verification of the IDEAS Building Energy Simulation Library. Journal 
of Building Performance Simulation, 11(6):669-688, 2018c. doi:10.1080/19401493.2018.1428361.

F. Jorissen, M. Wetter, and L. Helsen. Simplifications for Hydronic System Models in Modelica. Journal of Building Performance Simulation, 11(6):639-654, 2018d. doi:10.1080/19401493.2017.1421263.

D. Müller, M. Lauster, A. Constantin, M. Fuchs, and P. Remmen. AIXLIB - An Open-Source Modelica Library Within the IEA-EBC Annex 60 Framework. In J. Grunewald, C. Felsmann, A. Nicolai, and J. Seifert, editors, BauSIM, pages 3-9, Dresden, 2016. Fraunhofer IRB Verlag, Stuttgart.

C. Nytsch-Geusen, J. Huber, M. Ljubijankic, and J. Rädler. Modelica buildingsystems- eine modellbibliothek zur simulation komplexer energietechnischer gebäudesysteme. Bauphysik, 35(1):21-29, 2013.

D. Picard, F. Jorissen, and L. Helsen. Methodology for Obtaining Linear State Space Building Energy Simulation Models. In 11th International Modelica Conference, pages 51-58, Paris, France, 2015. doi:10.3384/ecp1511851.

M. Wetter. Modelica-based Modeling and Simulation to Support Research and Development in Building. Journal of Building Performance Simulation, 2(2):143-161, 2009.

M. Wetter. Fan And Pump Model That Has A Unique Solution For Any Boundary Condition And Control Signal. In 13th Conference of International Building Performance Simulation Association, pages 3505-3512, Chambéry, France, 2013.

M. Wetter and C. van Treeck. IEA EBC Annex 60: New Generation Computing Tools for Building and Community Energy Systems. The Regents of the University of California and RWTH Aachen University, 2017. ISBN 978-0-692-89748-5. URL http: / / www. iea-annex60.org/pubs.html.

M. Wetter, W. Zuo, T. S. Nouidui, and X. Pang. Modelica buildings library. Journal of Building Performance Simulation, 7(4):253-270, 2014. doi:DOI:10.1080/19401493.2013.765506.

M. Wetter, T. S. Nouidui, D. Lorenzetti, E. A. Lee, and A. Roth. Prototyping the Next Generation EnergyPlus Simulation Engine. In J. Mathur and V. Garg, editors, 14th Conference of International Building Performance Simulation Association, pages 403-410, Hyderabad, 2015. International Building Performance Simulation Association.

M. Wetter, M. Bonvini, and T. S. Nouidui. Equation-based languages - A new paradigm for building energy modeling, simulation and optimization. Energy \& Buildings, 117:290-300, 2016. doi:10.1016/j.enbuild.2015.10.017. 
